\title{
circASS1 overexpression inhibits the proliferation, invasion and migration of colorectal cancer cells by regulating the miR-1269a/VASH1 axis
}

\author{
HAI-LIN XIONG $^{1 *}$, XIAO-HUA ZHONG $^{2 *}$, XIAO-HONG GUO $^{1}$, HAO-JIE LIAO $^{1}$ and XIA YUAN ${ }^{1}$ \\ Departments of ${ }^{1}$ Medical Oncology and ${ }^{2}$ Gastroenterological Surgery, \\ Huizhou Municipal Central Hospital of Guangdong Province, Huizhou, Guangdong 516000, P.R. China
}

Received January 30, 2021; Accepted July 9, 2021

DOI: $10.3892 /$ etm.2021.10589

\begin{abstract}
Colorectal cancer (CRC), the third most common cancer worldwide, poses a threat to human life. However, its underlying mechanism is unclear and no satisfactory treatment is available. The present study aimed to investigate the role of circular RNA argininosuccinate synthase 1 (circASS1) in CRC cells and tissues to identify the potential mechanism underlying the pathogenesis of CRC. The expression of circASS1 in CRC cells and tissues was determined by reverse transcription-quantitative PCR. Following circASS1 overexpression in HT29 cells, cell viability, colony formation and apoptosis were measured using MTT, colony formation and TUNEL assays, respectively. Cell invasion and migration were also assessed. After confirming the associations among circASS1, microRNA (miR)-1269a and vasohibin 1 (VASH1), the characteristics of the HT29 cell line were assessed by performing the aforementioned assays. circASS1 expression was decreased in CRC cells and tissues, and circASS1 overexpression suppressed CRC cell proliferation, invasion and migration. circASS1 adsorbed miR-1269a and regulated its expression, and VASH1 was a target protein of miR-1269a. circASS1 overexpression decreased cell proliferation, invasion and migration, but enhanced cell apoptosis in HT29 cells, which was reversed by co-transfection with miR-1269a mimic or short hairpin RNA-VASH1. In conclusion, circASS1 overexpression inhibited CRC cell proliferation, invasion and migration by regulating miR-1269a/VASH1, which indicated
\end{abstract}

Correspondence to: Dr Xia Yuan, Department of Medical Oncology, Huizhou Municipal Central Hospital of Guangdong Province, 41 Eeling North Road, Huizhou, Guangdong 516000, P.R. China

E-mail: yuanxiayyx@163.com

*Contributed equally

Key words: proliferation, invasion, circular RNA argininosuccinate synthase 1/microRNA-1269a/vasohibin 1, migration, colorectal cancer a potential molecular mechanism underlying the pathogenesis of CRC.

\section{Introduction}

Colorectal cancer (CRC) is the third most common type of cancer worldwide and poses a threat to human life (1). Current advances in the treatment of CRC have not successfully decreased the high incidence of CRC (2). The pathogenesis of CRC is not completely understood; therefore, the identification of novel targets to effectively improved the severe pathological conditions of patients with CRC is important (3).

Vasohibin 1 (VASH1) is an endogenous inhibitor of angiogenesis induced by VEGF and fibroblast growth factor 2 (4). It has been reported that angiogenesis is inhibited by a negative feedback mechanism under physiological conditions (4). A study revealed that VASH1 was upregulated in several types of tumor tissues, and exhibited therapeutic efficacy by preventing tumor angiogenesis and suppressing tumor growth in a nude mice xenograft model (5). VASH1 is not only expressed in the vascular endothelium, but also in tumor cells (6,7). Another study demonstrated that microRNA (miR)-143-3p triggered by N6-methyladenosine promoted lung cancer brain metastasis via regulating VASH1 (8). In addition, VASH1 could not cause damage to healthy blood vessels in mice (9). Angiogenesis in hepatocellular carcinoma (HCC) could be promoted by the VEGF-mediated enhancer of zeste 2 polycomb repressive complex 2 subunit/VASH1 signaling pathway in cancer-related fibroblasts (10). VASH1 is primarily expressed in the cytoplasm of bladder cancer cells and several vascular endothelial cells. VASH1 was associated with tumor stage, pathological grade and distant metastasis, whereas its high expression was significantly associated with 5-year overall survival (OS) and progression free survival (PFS) rates (11). In CRC tissues, the expression levels of VASH1 and VEGFA were positively correlated with microvessel density. The OS and PFS of patients with high VASH1 expression were significantly lower compared with those with low VASH1 expression, which indicated that VASH1 protein expression is an independent risk factor affecting OS and PFS (7). VASH1 overexpression promoted CRC cell apoptosis and senescence, inhibited CRC 
cell proliferation and colony formation, and suppressed tumor growth in vivo (12).

miRNAs are non-coding endogenous conserved RNA molecules that bind to the 3'-untranslated regions of their target mRNAs $(13,14)$. Emerging evidence has suggested that miR-1269a is upregulated in CRC (15). A study showed that the expression of miR-1269a was increased in non-small cell lung cancer (NSCLC) tissues compared with that in adjacent healthy tissues, whereas miR-1269a knockdown attenuated NSCLC cell proliferation, colony formation and cell cycle progression (16). Another study suggested that miR-1269 could serve as an oncogene by promoting tumor metastasis and forming a positive feedback loop with TGF- $\beta$ (15). Additionally, miR-1269a overexpression could enhance the apoptosis of gastric cancer cells (17). Finally, long non-coding RNA LINC00261 could attenuate lung cancer tumor growth and metastasis via the miR-1269a/FOXO1 signaling pathway (18). Therefore, we speculated that miR-1269a may regulate $\mathrm{CRC}$ cells via targeting VASH1.

Circular RNAs (circRNAs/circs) are a class of endogenous non-coding RNAs with covalently closed continuous loops (19). A previous study reported that circASS1 expression is decreased and can inhibit the invasion and migration of breast cancer cells (20). The potential regulatory association between miR-1269a and circASS1, and how circASS1 regulates CRC cells via the miR-1269a/VASH1 axis is not completely understood.

Therefore, the present study aimed to elucidate the role of the circASS1/miR-1269a/VASH1 axis in regulating the proliferation, invasion and migration of CRC cells, as well as the potential underlying mechanism to identify novel therapeutic targets for CRC.

\section{Materials and methods}

Clinical specimens. Tumor tissue samples and pathologically verified normal tissue samples taken $>2 \mathrm{~cm}$ from the tumor were collected from 10 patients with CRC who were not treated with chemotherapy or radiotherapy from Huizhou Municipal Central Hospital of Guangdong Province (Huizhou, China) between May 2018 and May 2019. In total, seven males and three females were recruited (age, 35-72 years). The inclusion criteria were patients with only primary colon cancer. Patients who had received preoperative chemotherapy or radiotherapy were excluded. Samples were snap frozen in liquid nitrogen immediately after collection and stored at $-80^{\circ} \mathrm{C}$ until further analysis. All patients provided written informed consent prior to enrollment in the present study. The present study was approved by the Huizhou Municipal Central Hospital of Guangdong Province.

Cell culture. CRC cell lines (HCT116, Caco2,LoVo, SW480 and HT29) were obtained from Procell Life Science \& Technology Co., Ltd. The normal colon epithelial cell line (HIEC-6) was obtained from BioVector Science Lab, Inc. The HT-29 cell line was authenticated by STR. HIEC- 6 cells were cultured in DMEM (Invitrogen; Thermo Fisher Scientific, Inc.) supplemented with 10\% FBS (Gibco; Thermo Fisher Scientific, Inc.). CRC cell lines were cultured in DMEM (Invitrogen; Thermo Fisher Scientific, Inc.) supplemented with 10\% FBS, $100 \mathrm{U} / \mathrm{ml}$ penicillin and $100 \mu \mathrm{g} / \mathrm{ml}$ streptomycin (both from Gibco;
Thermo Fisher Scientific, Inc.) All cell lines were cultured in a humidified incubator at $37^{\circ} \mathrm{C}$ in a $5 \% \mathrm{CO}_{2}$ atmosphere.

Cell transfection. miR-1269a mimic (100 nM; cat. no. miR10005923-1-5) negative control (NC) mimic (100 nM; cat. no. miR1N0000001-1-5), miR-1269a inhibitor (100 nM; cat. no. miR20005923-1-5) and NC inhibitor (100 nM; cat. no. miR2N0000001-1-5) were purchased from Guangzhou RiboBio Co., Ltd. pcDNA3.1-circASS1 (Oe-circASS1; 100 nM), pcDNA3.1-NC (Oe-NC; $100 \mathrm{nM}$ ), short hairpin RNA (shRNA) against VASH1 (shRNA-VASH1; $100 \mathrm{nM}$ ) and shRNA-NC (100 $\mathrm{nM}$ ) plasmids were all constructed by Shanghai GenePharma Co., Ltd. When HT29 cells reached 60-80\% confluence, cell transfection was performed using Lipofectamine ${ }^{\circledR}$ 3000 (Invitrogen; Thermo Fisher Scientific, Inc.) for $24 \mathrm{~h}$ at $37^{\circ} \mathrm{C}$. At $48 \mathrm{~h}$ post-transfection, cells were used for subsequent experiments. Untreated HT29 cells were used as the control.

Reverse transcription-quantitative PCR (RT-qPCR). Total RNA was extracted from cells and tissues using TRIzol ${ }^{\circledR}$ (Invitrogen; Thermo Fisher Scientific, Inc.) according to the manufacturer's instructions. Subsequently, RNA was reverse transcribed into cDNA using the All-in-One miRNA First-Strand cDNA Synthesis kit (GeneCopoeia, Inc.) or the PrimeScript RT Reagent kit with gDNA Eraser (Takara Bio, Inc.) in accordance with the manufacturer's protocol. qPCR was performed using the SYBR Green PCR kit (GeneCopoeia, Inc.) on the StepOnePlus ${ }^{\mathrm{TM}}$ Real-Time PCR system (Applied Biosystems; Thermo Fisher Scientific, Inc.). The thermocycling conditions were as follows: $95^{\circ} \mathrm{C}$ for $30 \mathrm{sec}$, followed by 40 cycles of $95^{\circ} \mathrm{C}$ for $10 \mathrm{sec}$ and $60^{\circ} \mathrm{C}$ for $30 \mathrm{sec}$. U6 and GAPDH were used as internal controls for miRNA and mRNA expression, respectively. Additionally, internal $\beta$-actin was used to normalize circRNA expression. The $2^{-\Delta \Delta C q}$ method was used to determine relative expression (21). Primer sequences were as follows: circASS1 forward, 5'-GCCGTATTGACA TCGTGGAG-3' and reverse, 5'-TCGAGAATGTCAGGGGTG T-3'; miR1269a forward, 5'-GCTGGACTGAGCCGTGC-3' and reverse, 5'-CAGTGCGTGTCGTGGAGT-3'; VASH1 forward, 5'-AGATCCCCATACCGAGTGTG-3' and reverse, 5'-GCTTCCAGGCATTTGATTGGC-3'; U6 forward, 5'-AGC CCGCACTCAGAACATC-3' and reverse, 5'-GCCACCAAG ACAATCATCC-3'; GAPDH forward, 5'-ACAACTTTGGTA TCGTGGAAGG-3' and reverse, 5'-GCCATCACGCCACAG TTTC-3' and $\beta$-actin forward, 5'-CACCTTCTACAATGA GCTGCGTGTG-3' and reverse, 5'-ATAGCACAGCCTGGA TAGCAACGTAC-3'.

Western blotting. Total protein was extracted from cells using an RIPA lysis buffer kit (Boster Biological Technology). Protein concentrations were quantified using the bicinchoninic acid method (Thermo Fisher Scientific, Inc.). Following protein separation via 10\% SDS-PAGE (40 $\mu \mathrm{g} /$ lane), proteins were transferred onto PVDF membranes (Beyotime Institute of Biotechnology). Following blocking with 5\% skimmed milk for $2 \mathrm{~h}$ at room temperature, the membranes were then incubated overnight at $4^{\circ} \mathrm{C}$ with primary antibodies targeted against: VASH1 (cat. no. ab199732; 1:1,000; Abcam), Bcl-2 (cat. no. ab32124; 1:1,000; Abcam), Bax (cat. no. ab32503; 1:1,000; Abcam), cleaved caspase-3 (cat. no. ab32042; 1:500; 
Abcam), caspase-3 (cat. no. ab32351; 1:500; Abcam), cleaved poly (ADP-ribose) polymerase (PARP; cat. no. ab32064; 1:1,000; Abcam), PARP (cat. no. ab191217; 1:1,000; Abcam) and GAPDH (cat. no. ab9485; 1:2,500; Abcam). After washing three times with TBST (10\% Tween-20), the membranes were then incubated with the HRP-conjugated goat anti-rabbit secondary antibody (cat. no. ab6721; 1:2,000; Abcam) for $1 \mathrm{~h}$ at room temperature. The signals were developed using an enhanced chemiluminescence kit (EMD Millipore). Densitometric analysis was performed using ImageJ (version 1.48v; National Institutes of Health).

Colony formation assay. HT29 cells were seeded ( $2 \times 10^{3}$ cells/well) into 96 -well plates and cultured in complete medium until colony formation. After washing three times with PBS, the colonies were fixed with $100 \%$ methanol for $30 \mathrm{~min}$ at room temperature and stained with crystal violet at room temperature for $20 \mathrm{~min}$. Clones containing $\geq 50$ cells were counted. Cells were observed under a light microscope (DM1000; Leica Microsystems $\mathrm{GmbH}$ ) and five fields of view were randomly selected for manual counting of colonies.

MTT assay. Following transfection, HT29 cells were seeded ( $2 \times 10^{4}$ cells/well) into 96 -well plates for $24 \mathrm{~h}$. Subsequently, culture medium was discarded and serum-free medium was added. Then, $20 \mu \mathrm{l}$ MTT reagent $(5 \mathrm{mg} / \mathrm{ml})$ was added to each well and incubated for an additional $4 \mathrm{~h}$. The medium was discarded and $100 \mu \mathrm{l}$ dimethyl sulfoxide was added to each well to dissolve the purple formazan. The optical density was measured at a wavelength of $490 \mathrm{~nm}$ using a microplate reader (Bio-Rad Laboratories, Inc.).

TUNEL assay. Transfected HT29 cells were fixed with $4 \%$ paraformaldehyde for $30 \mathrm{~min}$ at room temperature. Subsequently, the TdT enzyme was added onto the slide and cells were incubated at $37^{\circ} \mathrm{C}$ for $2 \mathrm{~h}$. Cells were then incubated for an additional $1 \mathrm{~h}$ at room temperature in the dark in the presence of streptavidin-HRP. Then, $0.05 \%$ DAB was stained at room temperature for $5 \mathrm{~min}$, mounting medium (Beijing Solarbio Science \& Technology, Inc.) was used to seal the slides, and the cells were observed under a fluorescent microscope with 5 random fields of view per group. Washing with PBS was performed between all steps.

Matrigel invasion assays. To evaluate cell migration and invasion abilities, a Transwell chamber (Corning, Inc.) precoated with Matrigel at $37^{\circ} \mathrm{C}$ overnight was used. Transfected HT29 cells $\left(5 \times 10^{4}\right)$ were suspended in $200 \mu 1$ serum-free DMEM and then added to the upper chamber, the lower chamber was supplemented with DMEM containing 10\% FBS and incubated for $24 \mathrm{~h}$ at $37^{\circ} \mathrm{C}$. After washing three times with PBS, cells were fixed with $4 \%$ paraformaldehyde for $30 \mathrm{~min}$ at room temperature and then stained with $0.1 \%$ crystal violet solution for $15 \mathrm{~min}$ at room temperature and observed under a light microscope. Cell migration and invasion rates were determined based on crystal violet staining.

Wound healing assay. Following transfection, HT29 cells were grown to $100 \%$ confluence in 6 -well culture plates. Subsequently, a $10-\mu 1$ pipette tip was used to scratch the cell
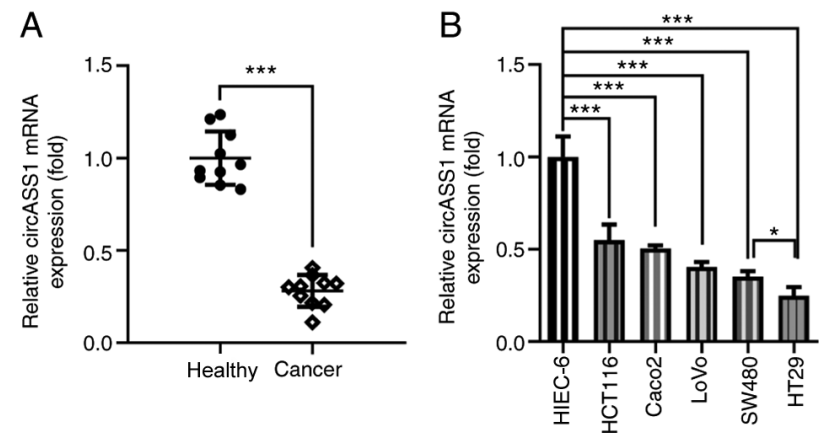

Figure 1. circASS1 is downregulated in CRC cells and tissues. Expression levels of circASS1 in CRC (A) tissues and (B) cell lines. ${ }^{*} \mathrm{P}<0.05$ and $^{* * * *} \mathrm{P}<0.001$. circASS1, circular RNA argininosuccinate synthase 1; CRC, colorectal cancer.

monolayer, followed by washing with PBS washing to remove cell debris. Cells were cultured in DMEM supplemented with $2 \%$ FBS. The wound was observed and photographed at 0 and $24 \mathrm{~h}$ using a light microscope with a digital camera and quantified using ImageJ (version $1.48 \mathrm{v}$; National Institutes of Health).

Luciferase reporter assay. The association between miR-1269a, VASH and circASS1 was predicted using the Encyclopedia of RNA Interactomes (ENCORI) database (starbase.sysu.edu.cn/index.php). The binding sequences of miR-1269a on circASS1 cDNA and VASH1 and their mutated antisense sequences were separately subcloned into the psiCHECK-2 vector (Promega Corporation). Subsequently, HT29 cells $\left(1 \times 10^{5}\right.$ cells/well) were co-transfected with the reporter plasmids $(100 \mathrm{nM})$ and $\mathrm{miR}-1269$ a mimic $(100 \mathrm{nM})$ or NC mimic (100 nM) using Lipofectamine 3000 (Invitrogen; Thermo Fisher Scientific, Inc.). Following incubation at $37^{\circ} \mathrm{C}$ for $48 \mathrm{~h}$, the firefly and Renilla luciferase activities were measured using the Renilla-Firefly Luciferase Dual Assay kit (Pierce, Thermo Fisher Scientific, Inc.).

Statistical analysis. Each experiment was repeated three times. Statistical analyses were performed using GraphPad Prism 6.0 software (GraphPad Software, Inc.). Comparisons between two groups were analyzed using paired Student's t test, whereas comparisons among multiple groups were analyzed using one-way ANOVA followed by Tukey's post hoc test. Pearson's correlation coefficient was used to determine correlations. $\mathrm{P}<0.05$ was considered to indicate a statistically significant difference. All data are presented as mean \pm standard deviation.

\section{Results}

circASS1 is downregulated in CRC cells and tissues. To elucidate the effect of circASS1 on CRC progression, its expression in CRC cells and tissues was determined by RT-qPCR. As shown in Fig. 1A, the expression levels of circASS1 were significantly decreased in CRC tissues compared with those in adjacent healthy tissues. Furthermore, circASS1 expression was significantly downregulated in CRC cells compared with that in HIEC-6 cells, with HT29 cells displaying the lowest circASS1 expression levels among all CRC cell lines. 

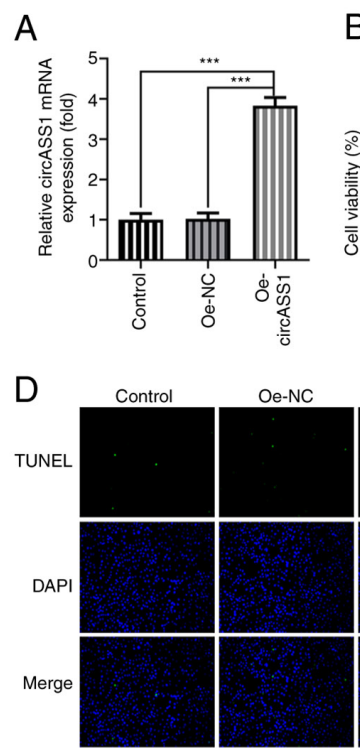

G
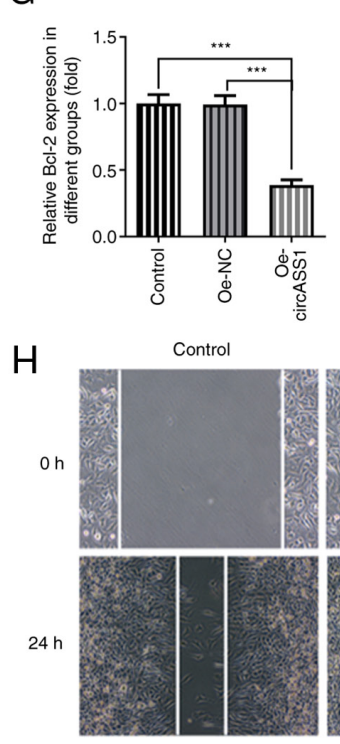

J

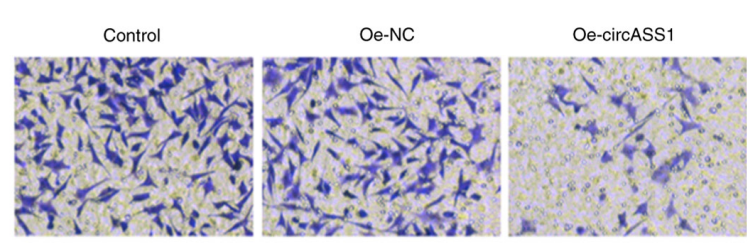

B

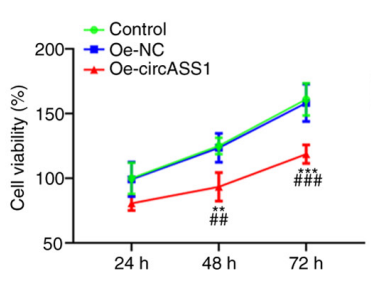

E

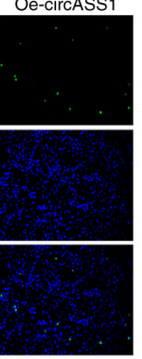

Oe-NC

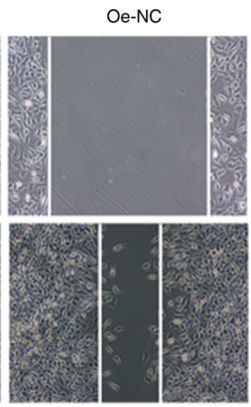

Oe-circASS1

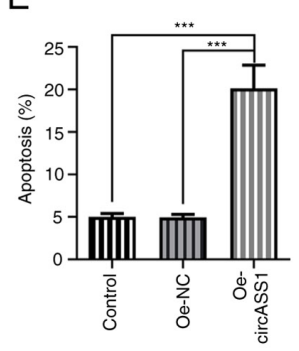

F
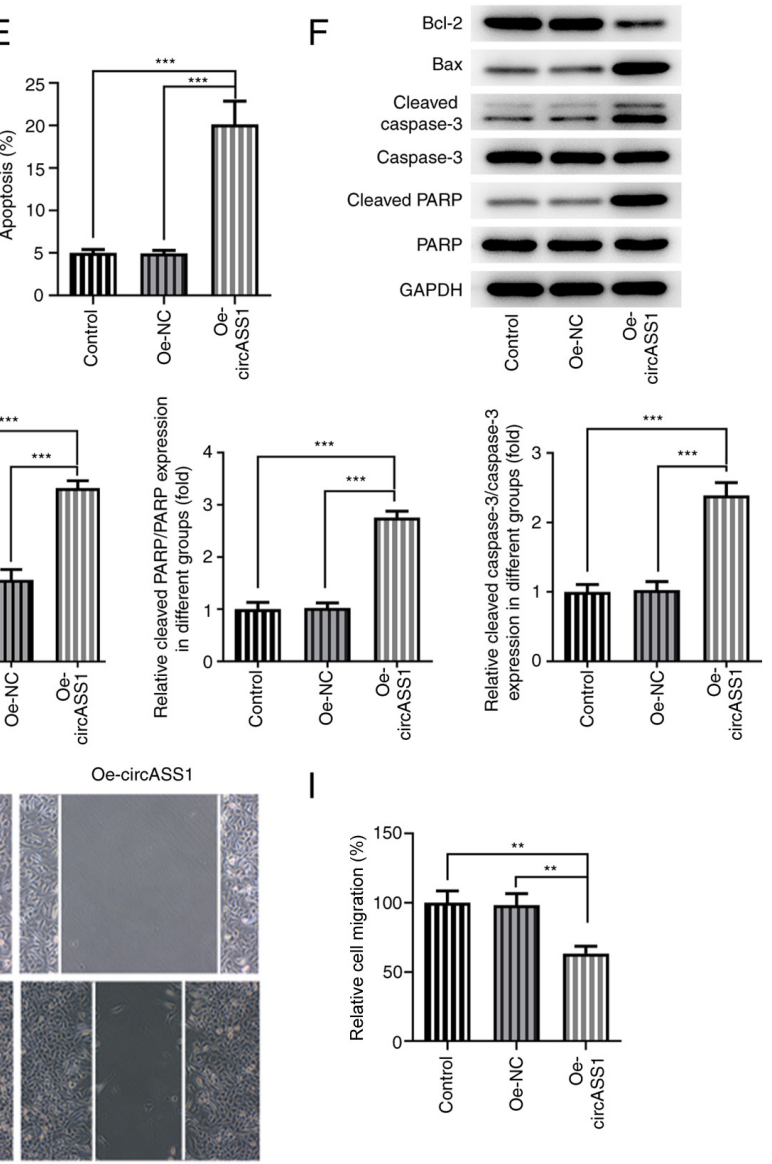

I
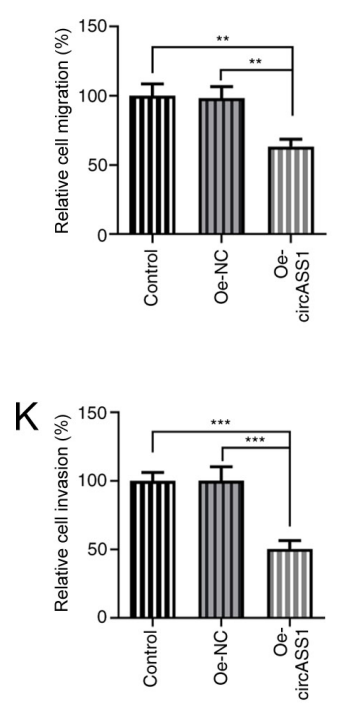

Figure 2. circASS1 overexpression suppresses colorectal cancer cell proliferation, invasion and migration. Following circASS1 overexpression in HT-29 cells, (A) circASS1 expression levels, (B) cell viability and (C) colony formation were assessed. Cell apoptosis was (D, 100x) determined by performing TUNEL assays and (E) quantified. (F) Apoptosis-related protein expression levels were determined by western blotting and (G) quantified. (H) Cell migration was assessed by performing wound healing assays and (I) quantified. Cell invasion was ( $\mathrm{J}$ ) assessed by performing Transwell invasion assays and (K) quantified. Magnification, $x 100 .{ }^{* *} \mathrm{P}<0.01$ and ${ }^{* * *} \mathrm{P}<0.001$ vs. Control; ${ }^{\# \#} \mathrm{P}<0.01$ and ${ }^{\# \#} \mathrm{P}<0.001$ vs. Oe-NC. circASS1, circular RNA argininosuccinate synthase 1 ; Oe, overexpression; NC, negative control; PARP, poly(ADP-ribose) polymerase.

Therefore, HT29 cells were selected for subsequent experiments.

circASS1 overexpression attenuates CRC cell proliferation, invasion and migration. To further examine the specific effects of circASS1 on CRC development, circASS1 overexpression plasmids were constructed and transfected into
HT29 cells (Fig. 2A). The results showed that cell viability and proliferation were decreased in HT29 cells following transfection with Oe-circASS1 compared with transfection with Oe-NC (Fig. 2B and C). Subsequently, TUNEL assays were performed to assess the effect of circASS1 overexpression on CRC cell apoptosis. Compared with the Oe-NC group, circASS1 overexpression significantly induced apoptosis in 
A

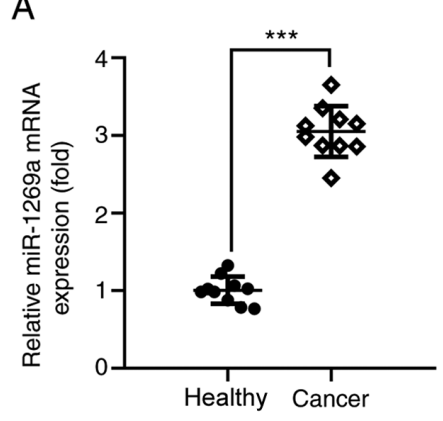

D

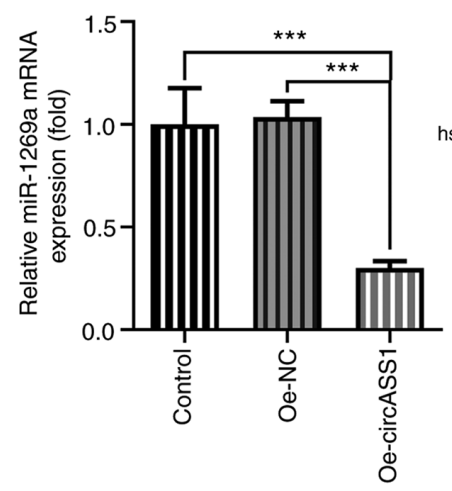

B

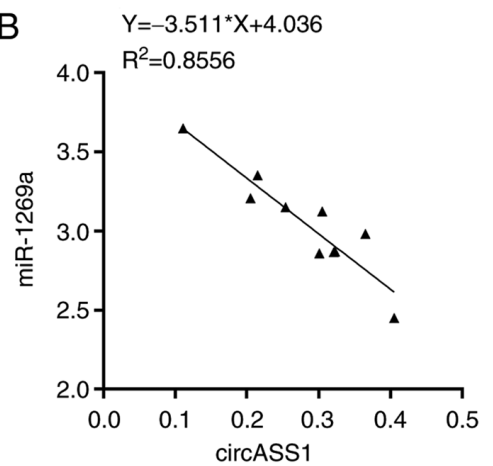

E

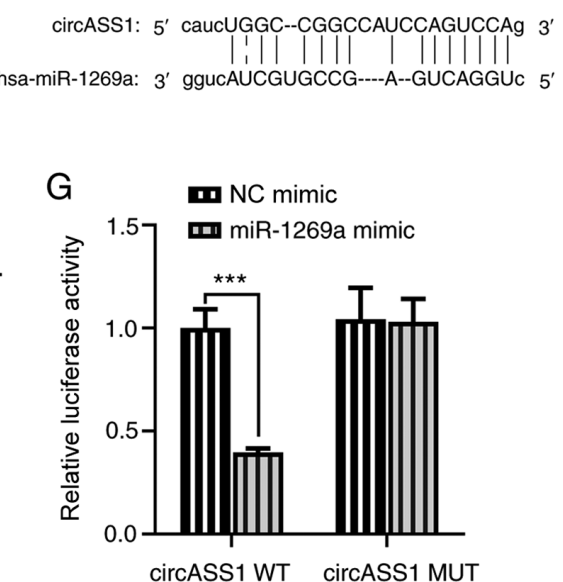

C

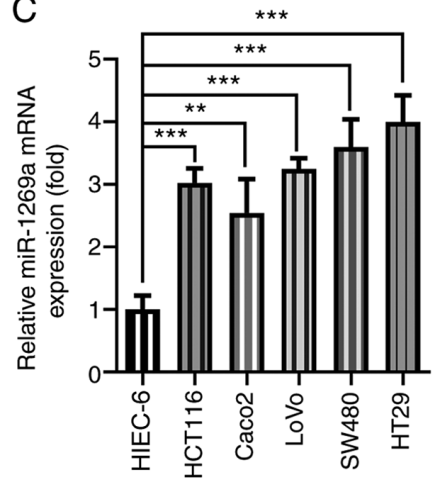

$\mathrm{F}$

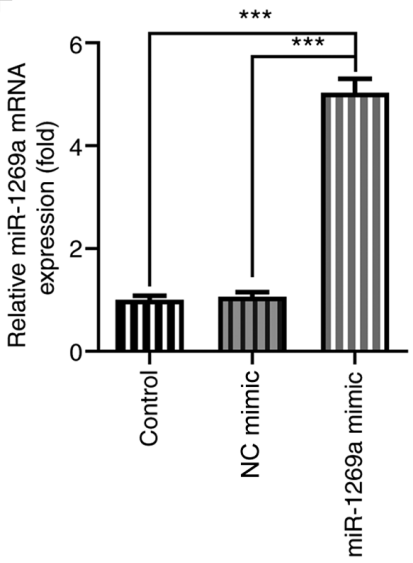

Figure 3. circASS1 adsorbs and negatively regulates miR-1269a expression. (A) miR-1269a expression levels in CRC tissues and adjacent healthy tissues. (B) Correlation between circASS1 and miR-1269a in CRC tissues. (C) miR-1269a expression levels in CRC cell lines and HIEC-6 cells. (D) Effect of circASS1 overexpression on miR-1269a expression in HT29 cells. (E) Binding sites between circASS1 and miR-1269a. (F) Transfection efficiency of miR-1269a mimic. (G) Luciferase reporter assays were performed to confirm the relationship between circASS1 and miR-1269a. ${ }^{* *} \mathrm{P}<0.01$ and ${ }^{* * *} \mathrm{P}<0.001$. circASS1, circular RNA argininosuccinate synthase 1; miR, microRNA; CRC, colorectal cancer; Oe, overexpression; NC, negative control; WT, wild-type; MUT, mutant.

HT29 cells (Fig. 2D and E). Moreover, compared with the Oe-NC group, circASS1 overexpression significantly decreased Bcl-2 expression and increased the expression of Bax, cleaved caspase-3/caspase-3 and cleaved PARP/PARP in HT29 cells (Fig. 2F). The invasion and migration abilities of HT29 cells were significantly inhibited after circASS1 overexpression in HT29 cells compared with the Oe-NC group (Fig. 2G-J). Taken together, the aforementioned findings indicated that circASS1 overexpression attenuated CRC cell proliferation, invasion and migration.

circASS1 adsorbs and negatively regulates miR-1269a. Previous studies have shown that miR-1269a is upregulated in CRC cells $(15,22)$. Consistently, miR-1269a expression was significantly increased in CRC tissue samples compared with that in adjacent healthy tissues (Fig. 3A). Correlation analysis confirmed the negative correlation between circASS1 and miR-1269a expression (Fig. 3B). Subsequently, miR-1269a expression levels were determined in different CRC cell lines and HIEC-6 cells. The results demonstrated that miR-1269a expression was significantly elevated in CRC cells compared with HIEC-6 cells, with HT29 cells displaying the highest miR-1269a expression levels (Fig. 3C). Following circASS1 overexpression in HT29 cells, miR-1269a expression was significantly downregulated compared with that in the Oe-NC group (Fig. 3D). The predicted binding sites between circASS1 and miR-1269a are presented in Fig. 3E. Subsequently, HT29 cells were transfected with miR-1269a mimic, and the association between circASS1 and miR-1269a was verified by performing the luciferase reporter assay (Fig. 3F and G). The results showed that co-transfection of circASS1 WT and miR-1269 significantly decreased the luciferase activity, demonstrating a negative correlation between the two. This indicated that circASS1 could adsorb and negatively regulate miR-1269a expression.

VASH1 is directly targeted by miR-1269a. The expression levels of VASH1 were evaluated by RT-qPCR and western blotting. As shown in Fig. 4A and B, VASH1 expression levels were significantly downregulated in CRC tissues compared with those in adjacent healthy tissues. Correlation coefficient analysis revealed a negative correlation between miR-1269a and VASH1, and a positive correlation between circASS1 and VASH1 in CRC tissue samples (Fig. 4C and D). VASH1 was also expressed at the lowest levels in HT29 cells among the CRC cell lines (Fig. 4E and F). Compared with NC inhibitor, transfection with miR-1269a inhibitor significantly decreased the expression levels of miR-1269a (Fig. 4G) and increased 

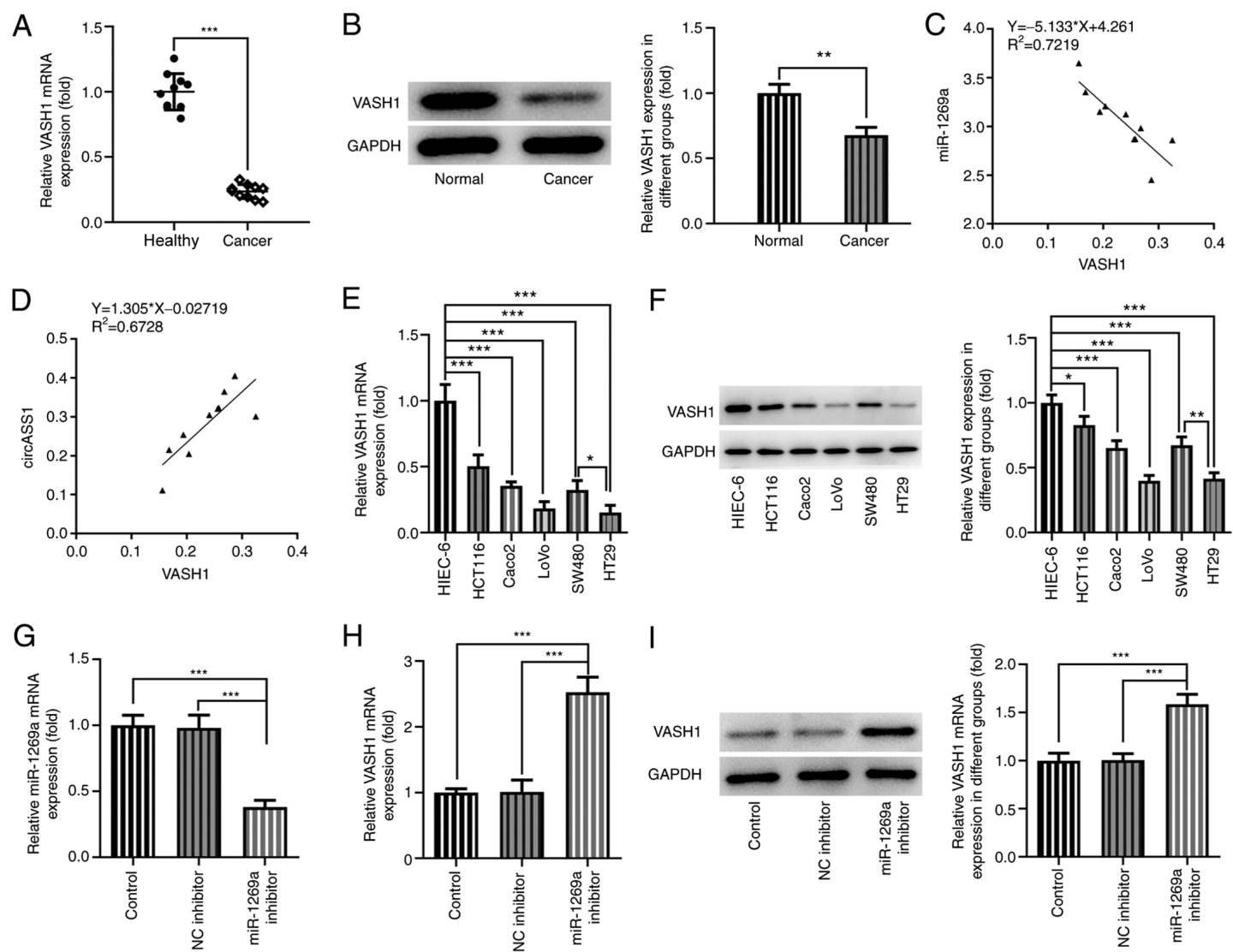

I
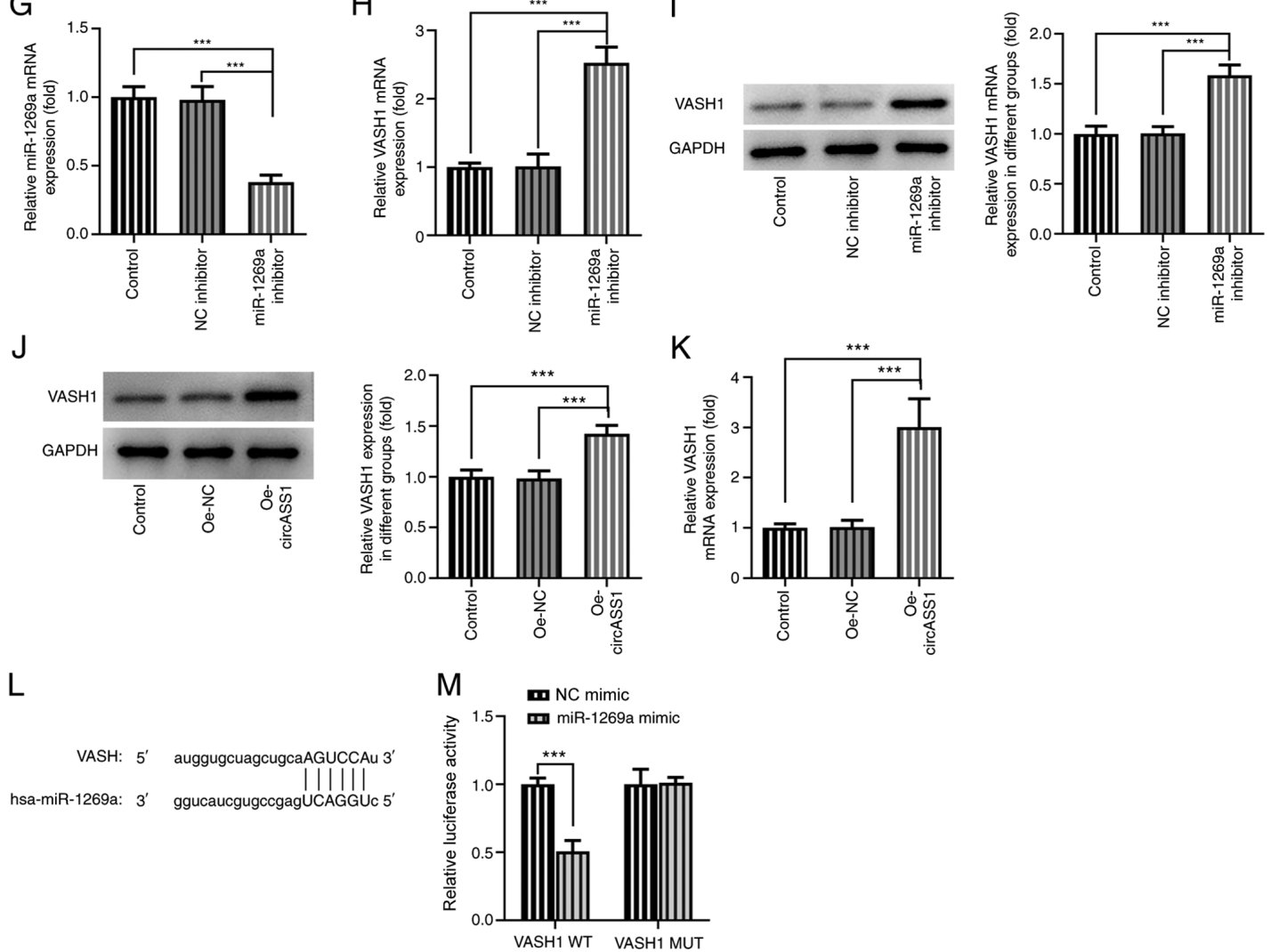

Figure 4. VASH1 is a target protein of miR-1269a. VASH1 (A) mRNA and (B) protein expression levels in CRC tissues and adjacent healthy tissues. Correlation between (C) miR-1269a and VASH1, and (D) circASS1 and VASH1 in CRC tissues. VASH1 (E) mRNA and (F) protein expression levels in CRC cell lines. (G) Transfection efficiency of miR-1269a inhibitor. VASH1 (H) mRNA and (I) protein expression levels in miR-1269a inhibitor-transfected HT29 cells. VASH1 (J) mRNA and (K) protein expression levels in Oe-circASS1-transfected HT29 cells. (J-K) The mRNA and protein levels of VASH1 in HT29 cells treated with Oe-circASS1. Binding sites between miR-1269a and VASH1 were (L) predicted and (M) confirmed. ${ }^{*} \mathrm{P}<0.05,{ }^{* * *} \mathrm{P}<0.01$ and ${ }^{* * * *} \mathrm{P}<0.001$. VASH1, vasohibin 1; miR, microRNA; CRC, colorectal cancer; Oe, overexpression; circASS1, circular RNA argininosuccinate synthase 1; NC, negative control; WT, wild-type; MUT, mutant.

the expression levels of VASH1 (Fig. 4H and I). Following transfection with Oe-circASS1, the expression of VASH1 was significantly upregulated compared with that in the Oe-NC group (Fig. 4J and K). Furthermore, a binding association between miR-1269a and VASH1 was predicted using ENCORI (Fig. 4L), and luciferase results showed that co-transfection of VASH1 WT and miR-1269 significantly decreased luciferase activity (Fig. 4M), confirming the binding association between
miR-1269a and VASH1. The results suggested that VASH1 was directly targeted by miR-1269a.

circASS1/miR-1269a/VASH1 axis enhances CRC cell proliferation. To investigate whether circASS1 could affect the characteristics of CRC cells via regulating the miR-1269a/VASH1 axis, VASH1 was knocked down for subsequent experiments (Fig. 5A and B). As shown in Fig. 5C and D, compared 

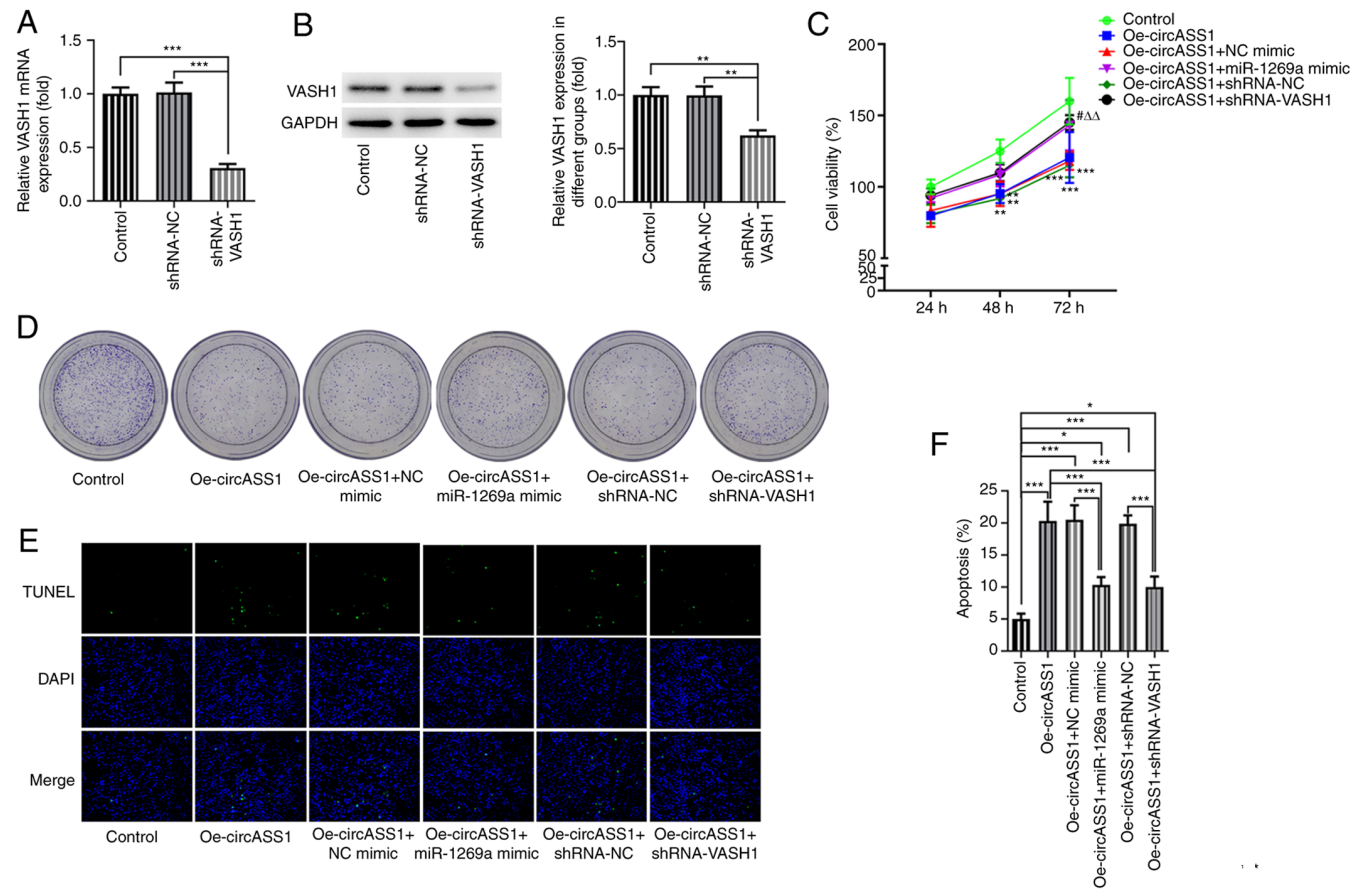

G
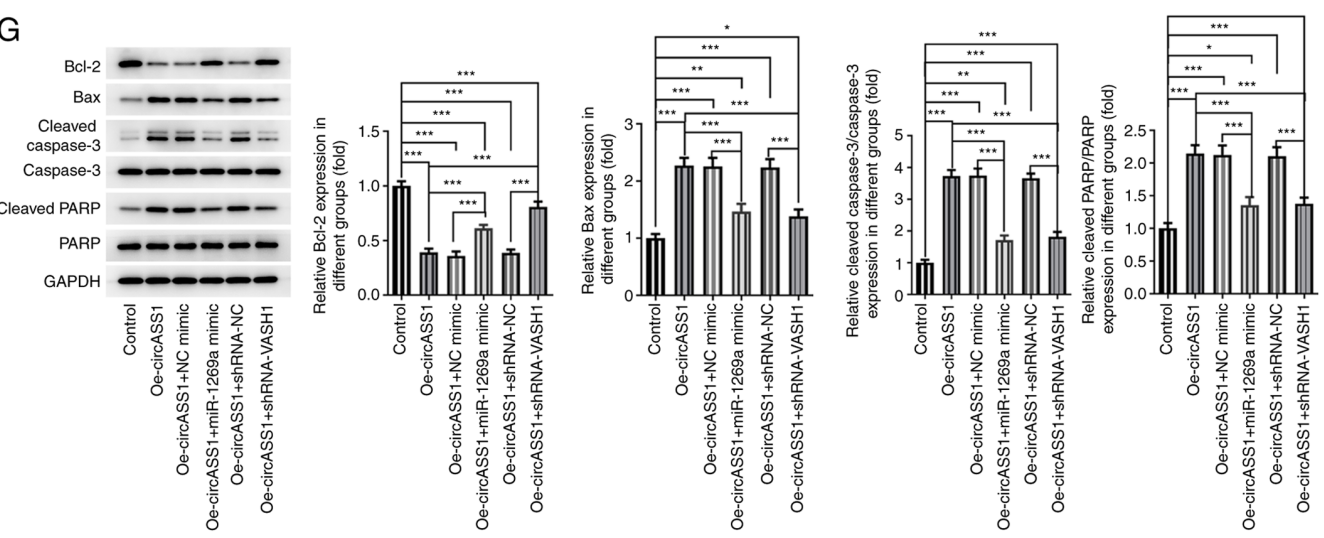

Figure 5. circASS1/miR-1269a/VASH1 axis enhances colorectal cancer cell proliferation. VASH1 (A) mRNA and (B) protein expression levels in shRNA-VASH1-transfected HT29 cells. (C) Cell viability and (D) colony formation of Oe-circASS1-transfected HT29 cells co-transfected with miR-1269a mimic or shRNA-VASH1. Cell apoptosis was (E) detected by performing TUNEL assays and (F) quantified. Magnification, x100. (G) Apoptosis-related protein expression levels were determined by western blotting. ${ }^{*} \mathrm{P}<0.05,{ }^{* *} \mathrm{P}<0.01$ and ${ }^{* * * *} \mathrm{P}<0.001$ vs. Control; ${ }^{*} \mathrm{P}<0.05$, Oe-circASS1+miR-1296a mimic vs Oe-circASS1+NC mimic; ${ }^{\Delta} \mathrm{P}<0.01$, Oe-circASS1+shRNA-VASH1 vs. Oe-circASS1+shRNA-NC. circASS1, circular RNA argininosuccinate synthase 1; miR, microRNA; VASH1, vasohibin 1; shRNA, short hairpin RNA; Oe, overexpression; NC, negative control; PARP, poly(ADP-ribose) polymerase.

with the control group, circASS1 overexpression attenuated HT29 cell viability and proliferation, which was abrogated by co-transfection with miR-1269a mimic or shRNA-VASH1. circASS1 overexpression-mediated HT29 cell apoptosis was significantly inhibited following co-transfection with miR-1269a mimic or shRNA-VASH1 (Fig. 5E and F). Compared with the control group, circASS1 overexpression significantly decreased Bcl-2 expression and increased the expression of Bax, cleaved caspase-3/caspase-3 and cleaved PARP/PARP in HT29 cells, which was significantly inhibited by co-transfection with miR-1269a mimic or shRNA-VASH1 (Fig. 5G).

circASS1/miR-1269a/VASH1 axis enhances CRC cell invasion and migration. Subsequently, the present study investigated the invasion and migration abilities of HT29 cells. Compared with the control group, circASS1 overexpression significantly decreased the migration and invasion abilities of HT29 cells, whereas co-transfection with miR-1269a mimic or shRNA-VASH1 significantly suppressed these effects (Fig. 6A and B). Therefore, these findings suggested that the circASS1/miR-1269a/VASH1 axis enhanced CRC cell proliferation, invasion and migration.

\section{Discussion}

The functions of circRNAs have been well documented (23). It has been reported that circRNAs interact with RNA binding proteins, serve as miRNA sponges and are involved in several biological processes, including transcriptional regulation and alternative splicing, indicating that circRNAs may participate in the development of various diseases $(24,25)$. Specifically, the role of circRNAs has been extensively investigated in multiple types of cancer (26-28). The unique characteristics of circRNAs highlight their potential as markers for the treatment of cancer (29). The tumor suppressive role of circASS1 in breast cancer has been previously reported (20). Therefore, 

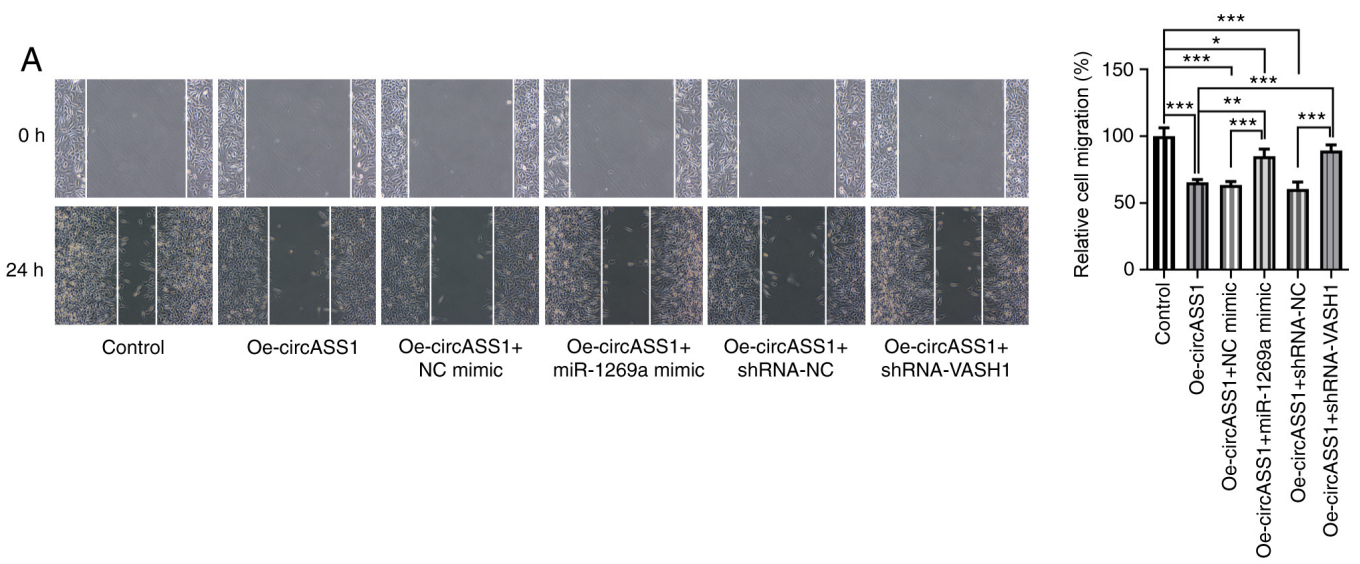

B

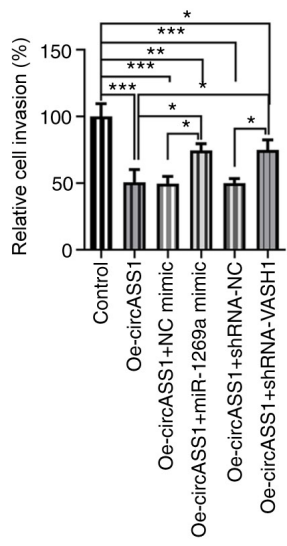

Figure 6. circASS1/miR-1269a/VASH1 axis enhances colorectal cancer cell invasion and migration. Cell (A) migration and (B) invasion of HT29 cells co-transfected with Oe-circASS1 and miR-1269a mimic or shRNA-VASH1. Magnification, $\mathrm{x} 100 .{ }^{*} \mathrm{P}<0.05,{ }^{* * *} \mathrm{P}<0.01$ and ${ }^{* * * *} \mathrm{P}<0.001$. circASS1, circular RNA argininosuccinate synthase 1; miR, microRNA; VASH1, vasohibin 1; Oe, overexpression; shRNA, short hairpin RNA; NC, negative control.

the present study hypothesized that circASS1 may also inhibit the progression of CRC via a certain mechanism. To the best of our knowledge, the present study was the first to demonstrate that circASS1 was downregulated in CRC tissues and cells. Furthermore, circASS1 overexpression inhibited CRC cell proliferation, invasion and migration, but enhanced cell apoptosis.

A plethora of genes have been identified as direct targets of miR-1269a. For instance, miR-1269a and its variant could affect the susceptibility and progression of HCC via targeting spermatogenesis associated serine rich 2 like and LDL receptor related protein 6 (30). miR-1269a also promoted NSCLC by regulating SOX6 (31). In the present study, miR-1269a was predicted to interact with circASS1. miR-1269a was reported to be expressed at abnormal levels in 14 types of cancer, suggesting that it could serve as a potential diagnostic marker for cancer treatment (32). A previous study demonstrated that the expression level of miR-1269a was increased in CRC and promoted CRC metastasis, which was further verified in the present study (15). The present study also indicated a negative correlation between miR-1269a and circASS1 expression.

VASH1, which was first recognized as a negative feedback modulator of angiogenesis, can regulate endothelial cell functions and further inhibit angiogenesis (33). VASH1 is not only expressed in endothelial cells but is also expressed in tumor cells (34). Therefore, further studies investigated whether this critical inhibitor of angiogenesis could serve as a key factor in tumor progression $(7,8,12)$. The potential value of
VASH1 in suppressing cancer cell proliferation and invasion has been confirmed by several studies, and antiangiogenic therapy is currently used for several types of cancer (35-37). Data have also demonstrated that VASH1 expression may inhibit tumorigenesis and metastasis in a human colon cancer model (12). Further associations between the clinicopathological features and VASH1 expression in patients with different types of cancer supported the potential use of VASH1 as a prognostic marker for cancer treatment $(38,39)$. The present study also indicated that VASH1 may prevent the progression of CRC by altering the cellular functions of CRC cells. VASH1 was expressed at relatively low levels in CRC tissue specimens compared with healthy tissue samples. Correlation analyses verified the negative correlation between miR-1269a and VASH1 expression, and a positive correlation between circASS1 and VASH1. Further assays demonstrated that miR-1269a inhibitor upregulated VASH1 in HT29 cells, and HT29 cells transfected with the circASS1 overexpression plasmid also displayed increased VASH1 expression. As the predicted direct association between miR-1269a and VASH1 was confirmed, the circASS1/miR-1269a/VASH1 axis was subsequently investigated to assess the potential mechanism underlying the effect of circASS1 on the biological functions of CRC cells. The results showed that circASS1 overexpression attenuated cell proliferation, invasion and migration, and enhanced the apoptosis of HT29 cells, and these effects were inhibited by co-transfection with miR-1269a mimic or shRNA-VASH1. 
In conclusion, the present study demonstrated that circASS1 overexpression inhibited CRC cell proliferation, invasion and migration by regulating the miR-1269a/VASH1 axis, thus providing a potential novel molecular mechanism involved in the pathogenesis of CRC.

\section{Acknowledgements}

Not applicable.

\section{Funding}

The present study was supported by the Medical Research Foundation of Guangdong Province (grant no. A2018240).

\section{Availability of data and materials}

The datasets used and/or analyzed during the current study are available from the corresponding author on reasonable request.

\section{Authors' contributions}

XHG collected clinical tissue samples. XY designed the experiments. HLX, XHZ, XHG and HJL performed the experiments. HLX and XHZ analyzed the data and wrote the manuscript. All authors read and approved the final manuscript. HLX and $\mathrm{XY}$ confirm the authenticity of all the raw data.

\section{Ethics approval and consent to participate}

All patients provided written informed consent prior to enrollment in the present study. The present study was approved by the Huizhou Municipal Central Hospital of Guangdong Province.

\section{Patient consent for publication}

Not applicable.

\section{Competing interests}

The authors declare they have no competing interests.

\section{References}

1. Bray F, Ferlay J, Soerjomataram I, Siegel RL, Torre LA and Jemal A: Global cancer statistics 2018: GLOBOCAN estimates of incidence and mortality worldwide for 36 cancers in 185 countries. CA Cancer J Clin 68: 394-424, 2018.

2. Siegel RL, Fedewa SA, Anderson WF, Miller KD, Ma J, Rosenberg PS and Jemal A: Colorectal cancer incidence patterns in the United States, 1974-2013. J Natl Cancer Inst 109, 2017.

3. Zha F, Qu X, Tang B, Li J, Wang Y, Zheng P, Ji T, Zhu C and Bai S: Long non-coding RNA MEG3 promotes fibrosis and inflammatory response in diabetic nephropathy via miR-181a/Egr-1/TLR4 axis. Aging (Albany NY) 11: 3716-3730, 2019.

4. Sato Y: The vasohibin family: A novel family for angiogenesis regulation. J Biochem 153: 5-11, 2013.

5. Li D, Zhou K, Wang S, Shi Z and Yang Z: Recombinant adenovirus encoding vasohibin prevents tumor angiogenesis and inhibits tumor growth. Cancer Sci 101: 448-452, 2010.

6. Zhao G, Yang Y, Tang Y, Han R and Sun Y: Reduced expression of vasohibin-1 is associated with clinicopathological features in renal cell carcinoma. Med Oncol 29: 3325-3334, 2012.
7. Yan Y, Shen Z, Ye Y, Jiang K, Zhang H, Shen C, Mustonen H, Puolakkainen P and Wang S: A novel molecular marker of prognosis in colorectal cancer: Vasohibin-1. Med Oncol 31: 816, 2014

8. Wang H, Deng Q, Lv Z, Ling Y, Hou X, Chen Z, Dinglin X, Ma S, $\mathrm{Li} \mathrm{D}, \mathrm{Wu}$ Y, et al: N6-methyladenosine induced miR-143-3p promotes the brain metastasis of lung cancer via regulation of VASH1. Mol Cancer 18: 181, 2019.

9. Heishi T, Hosaka T, Suzuki Y, Miyashita H, Oike Y, Takahashi T, Nakamura T, Arioka S, Mitsuda Y, Takakura T, et al: Endogenous angiogenesis inhibitor vasohibin1 exhibits broad-spectrum antilymphangiogenic activity and suppresses lymph node metastasis. Am J Pathol 176: 1950-1958, 2010.

10. Huang B, Huang M and Li Q: Cancer-associated fibroblasts promote angiogenesis of hepatocellular carcinoma by VEGF-Mediated EZH2/VASH1 pathway. Technol Cancer Res Treat 18: 1533033819879905, 2019.

11. Zhang B, Wu Z, Xie W, Tian D, Chen F, Qin C, Du Z, Tang G, Gao Q, Qiu X, et al: The expression of vasohibin-1 and its prognostic significance in bladder cancer. Exp Ther Med 14: 3477-3484, 2017.

12. Liu S, Han B, Zhang Q, Dou J, Wang F, Lin W, Sun Y and Peng G: Vasohibin-1 suppresses colon cancer. Oncotarget 6: 7880-7898, 2015.

13. Shukla GC, Singh J and Barik S: MicroRNAs: Processing, maturation, target recognition and regulatory functions. Mol Cell Pharmacol 3: 83-92, 2011.

14. Zhang Y, Wang Q, Luo N, Liu J, Ren H, Shao X, Zhang L and Yu Y: MicroRNA-1269a promotes proliferation and arrest of apoptosis of glioma cells by directly targeting ATRX. Front Oncol 10: 563901, 2020.

15. Bu P, Wang L, Chen KY, Rakhilin N, Sun J, Closa A, Tung KL, King S, Kristine Varanko A, Xu Y, et al: miR-1269 promotes metastasis and forms a positive feedback loop with TGF- $\beta$. Nat Commun 6: 6879, 2015.

16. Jin RH, Yu DJ and Zhong M: MiR-1269a acts as an onco-miRNA in non-small cell lung cancer via down-regulating SOX6. Eur Rev Med Pharmacol Sci 22: 4888-4897, 2018.

17. Li W, Zhang H, Min P, Zhu J, Xu D, Jiang W, Ma Y, Qiu J, $\mathrm{Xu}$ W, Chen J, et al: Downregulated miRNA-1269a variant (rs73239138) decreases the susceptibility to gastric cancer via targeting ZNF70. Oncol Lett 14: 6345-6354, 2017.

18. Guo C, Shi H, Shang Y, Zhang Y, Cui J and Yu H: LncRNA LINC00261 overexpression suppresses the growth and metastasis of lung cancer via regulating miR-1269a/FOXO1 axis. Cancer Cell Int 20: 275, 2020.

19. Memczak S, Jens M, Elefsinioti A, Torti F, Krueger J, Rybak A, Maier L, Mackowiak SD, Gregersen LH, Munschauer M, et al: Circular RNAs are a large class of animal RNAs with regulatory potency. Nature 495: 333-338, 2013.

20. Hou JC, Xu Z, Zhong SL, Zhang HD, Jiang LH, Chen X, Zhu LP, Li J, Zhou SY, Yang SJ, et al: Circular RNA circASS1 is downregulated in breast cancer cells MDA-MB-231 and suppressed invasion and migration. Epigenomics 11: 199-213, 2019.

21. Livak KJ and Schmittgen TD: Analysis of relative gene expression data using real-time quantitative PCR and the 2(-Delta Delta C(T)) method. Methods 25: 402-408, 2002.

22. Zhang J, Luo X, Li H, Deng L and Wang Y: Genome-wide uncovering of STAT3-mediated miRNA expression profiles in colorectal cancer cell lines. Biomed Res Int 2014: 187105, 2014.

23. Li XN, Wang ZJ, Ye CX, Zhao BC, Li ZL and Yang Y: RNA sequencing reveals the expression profiles of circRNA and indicates that circDDX17 acts as a tumor suppressor in colorectal cancer. J Exp Clin Cancer Res 37: 325, 2018.

24. Han B, Chao J and Yao H: Circular RNA and its mechanisms in disease: From the bench to the clinic. Pharmacol Ther 187: 31-44, 2018.

25. Liu J, Liu T, Wang $X$ and $\mathrm{He}$ A: Circles reshaping the RNA world: From waste to treasure. Mol Cancer 16: 58, 2017.

26. Zhang HD, Jiang LH, Sun DW, Hou JC and Ji ZL: CircRNA: A novel type of biomarker for cancer. Breast Cancer 25: 1-7, 2018.

27. Zhang T, Wu DM, Deng SH, Han R, Liu T, Li J and Xu Y: RNAseq profiling of circRNA expression in radiation-treated A549 cells and bioinformatics analysis of radiation-related circRNA-miRNA networks. Oncol Lett 20: 1557-1566, 2020.

28. Patop IL and Kadener S: circRNAs in Cancer. Curr Opin Genet Dev 48: 121-127, 2018.

29. Xu H, Liu Y, Cheng P, Wang C, Liu Y, Zhou W, Xu Y and Ji G: CircRNA_0000392 promotes colorectal cancer progression through the miR-193a-5p/PIK3R3/AKT axis. J Exp Clin Cancer Res 39: 283, 2020. 
30. Min P, Li W, Zeng D, Ma Y, Xu D, Zheng W, Tang F, Chen J, Shi J,Hu H, et al: A single nucleotide variant in microRNA-1269a promotes the occurrence and process of hepatocellular carcinoma by targeting to oncogenes SPATS2L and LRP6. Bull Cancer 104: 311-320, 2017.

31. Liu J and Dong YJ: Myocardial protective effect and mechanism of dexmedetomidine on myocardial ischemia-reperfusion injury in rats. Journal of Clinical and Experimental Medicine 17: 2476-2479, 2018 (In Chinese)

32. Hu Y, Dingerdissen H, Gupta S, Kahsay R, Shanker V, Wan Q, Yan C and Mazumder R: Identification of key differentially expressed MicroRNAs in cancer patients through pan-cancer analysis. Comput Biol Med 103: 183-197, 2018.

33. Miyashita $\mathrm{H}$, Watanabe T, Hayashi $\mathrm{H}$, Suzuki Y, Nakamura $\mathrm{T}$, Ito S, Ono M, Hoshikawa Y, Okada Y, Kondo T and Sato Y: Angiogenesis inhibitor vasohibin-1 enhances stress resistance of endothelial cells via induction of SOD2 and SIRT1. PLoS One 7: e46459, 2012.

34. Shimizu K, Watanabe K, Yamashita H, Abe M, Yoshimatsu H, Ohta $\mathrm{H}$, Sonoda $\mathrm{H}$ and Sato $\mathrm{Y}$ : Gene regulation of a novel angiogenesis inhibitor, vasohibin, in endothelial cells. Biochem Biophys Res Commun 327: 700-706, 2005.

35. Takahashi Y, Saga Y, Koyanagi T, Takei Y, Machida S, Taneichi A, Mizukami H, Sato Y, Matsubara S and Fujiwara H: The angiogenesis regulator vasohibin-1 inhibits ovarian cancer growth and peritoneal dissemination and prolongs host survival. Int J Oncol 47: 2057-2063, 2015.
36. Zhao G, Na R, Li L, Xiao H, Ding N, Sun Y and Han R: Vasohibin-1 inhibits angiogenesis and suppresses tumor growth in renal cell carcinoma. Oncol Rep 38: 1021-1028, 2017.

37. Zhang T, Jing L, Li H, Ding L, Ai D, Lyu J and Zhong L: MicroRNA-4530 promotes angiogenesis by targeting VASH1 in breast carcinoma cells. Oncol Lett 14: 111-118, 2017.

38. Tamaki K, Moriya T, Sato Y, Ishida T, Maruo Y, Yoshinaga K, Ohuchi N and Sasano H: Vasohibin-1 in human breast carcinoma: A potential negative feedback regulator of angiogenesis. Cancer Sci 100: 88-94, 2009.

39. Kanomata N, Sato Y, Miyaji Y, Nagai A and Moriya T: Vasohibin-1 is a new predictor of disease-free survival in operated patients with renal cell carcinoma. J Clin Pathol 66: 613-619, 2013.

(i) $($ This work is licensed under a Creative Commons Attribution-NonCommercial-NoDerivatives 4.0 International (CC BY-NC-ND 4.0) License. 